PROCEEDINGS OF THE

AMERICAN MATHEMATICAL SOCIETY

Volume 127, Number 6, Pages 1847-1854

S 0002-9939(99)04753-X

Article electronically published on February 17, 1999

\title{
REMARKABLE ASYMMETRIC RANDOM WALKS
}

\author{
L. MATTNER
}

(Communicated by Stanley Sawyer)

\begin{abstract}
There exists an asymmetric probability measure $P$ on the real line with $P^{* n}(] 0, \infty[)+(1 / 2) P^{* n}(\{0\})=1 / 2$ for every $n \in \mathbf{N}$. $P$ can be chosen absolutely continuous and $P$ can be chosen to be concentrated on the integers. In both cases, $P$ can be chosen to have moments of every order, but $P$ cannot be determined by its moments.
\end{abstract}

\section{Questions AND Results}

Let $P$ be a probability measure on the real line $\mathbf{R}$, let $P^{* n}$ denote its $n$th convolution power, with $n \in \mathbf{N}=\{1,2, \ldots\}$, and consider the following two properties that $P$ might have.

(S) $P$ is symmetric with respect to sign change.

(WS) $P^{* n}(] 0, \infty[)+(1 / 2) P^{* n}(\{0\})=1 / 2$ for every $n \in \mathbf{N}$.

If the symmetry condition (S) holds, then $P^{* n}$ is symmetric for every $n \in \mathbf{N}$, so that the weak symmetry condition (WS) trivially follows.

Question. Under what restrictions on $P$ does (WS) imply (S)?

A partial answer to this question has been given in [6, Theorem 2]. That paper also motivates the question by statistical estimation theory ["When is the sample mean a median unbiased estimator?"] and by random walk theory ["When has a random walk the same first positive entrance time distribution as a symmetric random walk?"]. The present paper provides a more complete answer.

It turns out that (WS) implies (S) under certain additional conditions but, surprisingly, not so without. In order to state this result precisely, let us consider the following further properties $P$ might have.

(C) The characteristic function $\varphi$ of $P$ satisfies $\lim \sup _{|t| \rightarrow \infty}|\varphi(t)|<1$.

(L) $P$ is concentrated on $a+h \mathbf{Z}$ for some $a \in \mathbf{R}$ and some $h \in] 0, \infty[$.

(D) $P$ is determined by its moments.

(M) $P$ possesses moments of every order.

Here (C) stands for Cramér, (L) for lattice, (D) for determinateness, and (M) for moments. It is well known that (D) implies (M) but not vice versa.

The following theorem summarizes our present knowledge about the question. [The contributions of this paper are stated more explicitly in Section 2 below, as

Received by the editors May 5, 1997 and, in revised form, September 22, 1997.

1991 Mathematics Subject Classification. Primary 60J15, 60E10, 62E10, 62 G05.

Key words and phrases. Characteristic function, characterization of symmetry, Edgeworth expansion, Gurland inversion, median unbiased estimator.

(C)1999 American Mathematical Society 
Propositions 1 and 2, and as Proposition 3, parts ii), iii), iv), the latter part read under the assumption (L).]

Theorem. The following statement is true under each of the two assumptions (C) or $(\mathrm{L})$ :

(WS) and (D) jointly imply (S), but (WS) and (M) do not.

Observe that the theorem says nothing about probability measures satisfying neither $(\mathrm{C})$ nor $(\mathrm{L})$. Hence the following subquestion remains open.

Subquestion. Does (S) already follow from (WS) and (D), that is, without assuming $(\mathrm{C})$ or $(\mathrm{L})$ ?

As a first step towards an affirmative answer to the subquestion, one could try to prove part iv) of Proposition 3 in the case $k=2$, without assuming (C) or (L).

Remark. Though not strictly related to the results of the present paper, we would like to mention here that Mattner [7] acknowledges partial priority for Theorem 1 of Mattner [6] to Pollak [10].

\section{Proofs}

Proof of the theorem. Assume (C). Then (WS) and (D) imply (S) by Theorem 2 of Mattner [6]. And the Riemann-Lebesgue lemma applied to a probability measure $P$ given by Proposition 1 , stated and proved below, shows that (WS) and (M) do not imply $(\mathrm{S})$.

Now assume (L) instead of (C). Then (WS) and (D) easily imply (S) via part iv) of Proposition 3. And Proposition 2 shows that (WS) and (M) do not imply (S).

Proposition 1. There exists an absolutely continuous probability measure $P$ on the real line which satisfies (WS) and $(\mathrm{M})$ but not $(\mathrm{S})$.

Proof. If $\mu$ is any complex measure on the real line $\mathbf{R}$ with finite total variation, and if $\widehat{\mu}$ denotes its Fourier transform defined by $\widehat{\mu}(t)=\int \exp (i t x) d \mu(x)$ for $t \in \mathbf{R}$, then an inversion theorem of Gurland [5] states that

(1) $\mu(]-\infty, x[)+\frac{1}{2} \mu(\{x\})=\frac{\widehat{\mu}(0)}{2}+\frac{1}{2 \pi}$ V.P. $\int_{-\infty}^{\infty} \widehat{\mu}(t) \frac{e^{-i t x}}{-i t} d t \quad(x \in \mathbf{R})$,

with the existence of the principal value integral V.P. $\int_{-\infty}^{\infty}=\lim _{\varepsilon \downarrow}, A \uparrow \infty \int_{-A}^{-\varepsilon}+\int_{\varepsilon}^{A}$ being part of the conclusion.

If now $P$ is a probability measure on $\mathbf{R}$ with Fourier transform [characteristic function] $\varphi$, then, using hermiteness, $(\mathrm{S})$ is seen to be equivalent to

$(\widehat{\mathrm{S}}) \operatorname{Im} \varphi(t)=0$ for every $t \in] 0, \infty[$,

and, using in addition (1) applied to $\mu=P^{* n}$ and $x=0$, (WS) is seen to be equivalent to

$(\widehat{\mathrm{WS}}) \int_{\downarrow 0}^{\uparrow \infty} \operatorname{Im} \varphi^{n}(t) \frac{d t}{t}=0$ for every $n \in \mathbf{N}$.

Finally, (M) is equivalent to

$(\widehat{M}) \varphi$ is infinitely often differentiable. 
So we have to construct a characteristic function of an absolutely continuous probability measure which satisfies $(\widehat{\mathrm{WS}})$ and $(\widehat{\mathrm{M}})$, but not $(\widehat{\mathrm{S}})$.

We start as in [6, Proof of Theorem 3]: Let $\varepsilon, a \in] 0, \infty[$ with

$$
3 \varepsilon<a .
$$

Let $\psi$ be a real-valued and infinitely often differentiable characteristic function with support contained in $[-\varepsilon, \varepsilon]$. [The existence of such a function is well known. See, for example, page 184 of Donoghue [1] for the classical construction. The proof of Proposition 1 in Ramachandran [11] provides an alternative one.] Put

$$
\varphi_{1}(t):=\psi(t)+\frac{i}{2} \psi(t-a)-\frac{i}{2} \psi(t+a) \quad(t \in \mathbf{R}) .
$$

Then $\varphi_{1}$ is a characteristic function. [If $g$ denotes a density corresponding to $\psi$, then $\varphi_{1}$ is the Fourier transform of the function

$$
f_{1}(x)=g(x) \cdot(1+(i / 2)(\exp (-i a x)-\exp (i a x))=g(x) \cdot(1+\sin a x) \geq 0,
$$

which integrates to one by $\varphi_{1}(0)=1$.]

Now the new and crucial idea is to define the characteristic function $\varphi$ as the average of $\varphi_{1}$ and a suitable reflected and scaled version of $\varphi_{1}$ as follows:

$$
\begin{aligned}
\varphi_{2}(t) & :=\varphi_{1}(2 t) \quad(t \in \mathbf{R}), \\
\varphi(t) & :=\frac{1}{2}\left(\varphi_{1}(t)+\varphi_{2}(-t)\right) \quad(t \in \mathbf{R}) .
\end{aligned}
$$

Then $\varphi$ is a characteristic function of an absolutely continuous probability measure, satisfying $(\widehat{\mathrm{M}})$. Writing $\varphi_{A}$ for the function $t \mapsto \varphi(t) 1_{A}(t)$ and using (2), we may decompose $\varphi_{0, \infty}$ into three disjointly supported summands as follows:

$$
\begin{aligned}
\varphi_{] 0, \infty[}(t) & =\varphi_{] 0, \varepsilon[}(t)+\varphi_{](a-\varepsilon) / 2,(a+\varepsilon) / 2[}(t)+\varphi_{] a-\varepsilon, a+\varepsilon[}(t) \\
& =\frac{1}{2}(\psi(t)+\psi(2 t))-\frac{i}{4} \psi(2 t-a)+\frac{i}{4} \psi(t-a) .
\end{aligned}
$$

[When checking the last term, remember that $\psi$, being a real-valued characteristic function, is even.] Now $\varphi_{]}(a-\varepsilon) / 2,(a+\varepsilon) / 2\left[+\varphi_{] a-\varepsilon, a+\varepsilon[}\right.$ is purely imaginary and not identically zero. Hence $(\widehat{\mathrm{S}})$ is not true.

To check that $(\widehat{\mathrm{WS}})$ is true, assume first that $n$ is an even positive integer. Since $\varphi(] 0, \infty[) \subset \mathbf{R} \cup i \mathbf{R}$ for our $\varphi$, the function $\varphi^{n}$ is real-valued. Hence the equation in $(\widehat{\mathrm{WS}})$ is trivially true in this case. Now assume that $n$ is odd. Then

$$
\begin{aligned}
\frac{4^{n}}{i^{n-1}} \int_{\downarrow 0}^{\uparrow \infty} \operatorname{Im} \varphi^{n}(t) \frac{d t}{t} & =-\int_{0}^{\infty} \psi^{n}(2 t-a) \frac{d t}{t}+\int_{0}^{\infty} \psi^{n}(t-a) \frac{d t}{t} \\
& =0,
\end{aligned}
$$

by scale-invariance of $d t / t$, so that the equation in $(\widehat{\mathrm{WS}})$ is also true in this case.

Proposition 2. There exists a probability measure $P$ concentrated on the integers which satisfies (WS) and (M) but not (S).

Proof. We continue to use the notation of the proof of Proposition 1, but we assume in addition that

$$
a+\varepsilon<2 \pi
$$


Put

$$
\begin{aligned}
\omega_{1}(t) & :=\sum_{k \in \mathbf{Z}} \varphi_{1}(t-2 \pi k) \quad(t \in \mathbf{R}), \\
\omega_{2}(t) & :=\omega_{1}(2 t) \quad(t \in \mathbf{R}), \\
\omega(t) & :=\frac{1}{2}\left(\omega_{1}(t)+\omega_{2}(-t)\right) \quad(t \in \mathbf{R}) .
\end{aligned}
$$

Then $\omega$ is the characteristic function of a probability measure concentrated on the integers by [3, p. 631 , Corollary], and $\omega$ satisfies $(\widehat{\mathrm{M}})$, but not $(\widehat{\mathrm{S}})$.

So it remains to prove (WS). Since $\omega$ is an infinite sum, the argument analogous to the one leading to $(3)$ and $(4)$, and thus to ( $\widehat{\mathrm{WS}})$, is a bit messy. It appears to be more elegant to use the following result.

Claim. If $\mu$ is any complex measure on the integers $\mathbf{Z}$ with finite total variation, and if $\widehat{\mu}$ denotes its Fourier transform defined by $\widehat{\mu}(t)=\int \exp (i t x) d \mu(x)$ for $t \in \mathbf{R}$, then

(6)

$$
\mu(]-\infty, x[)+\frac{1}{2} \mu(\{x\})=\frac{\widehat{\mu}(0)}{2}+\frac{1}{2 \pi} \text { V.P. } \int_{-\pi}^{\pi} \widehat{\mu}(t) \frac{e^{-i t x} d t}{-i 2 \tan (t / 2)} \quad(x \in \mathbf{Z}),
$$

with the existence of the principal value integral V.P. $\int_{-\pi}^{\pi}=\lim _{\varepsilon \downarrow 0} \int_{-\pi}^{-\varepsilon}+\int_{\varepsilon}^{\pi}$ being part of the conclusion.

[This claim can be proved by decomposing the integral $\int_{-\infty}^{\infty}$ on the right-hand side of (1) as $\sum_{n \in \mathbf{Z}} \int_{-\pi+n 2 \pi}^{\pi+n 2 \pi}$, then transforming every integral to one over $[-\pi, \pi]$, and finally using the partial fraction expansion of the cotangens. See Mattner [8] for a detailed proof and for further information on Gurland's inversion formula.]

Using (6), we see that (WS) for a $P$ concentrated on the integers, with characteristic function $\varphi$, is equivalent to

$\left(\widehat{\mathrm{WS}}_{\mathbf{Z}}\right) \int_{\downarrow 0}^{\pi} \operatorname{Im} \varphi^{n}(t) \cot (t / 2) d t=0$ for every $n \in \mathbf{N}$.

Using (2) and (5), we may decompose $\omega_{] 0, \pi]}$ into five disjointly supported summands as follows:

$$
\begin{aligned}
& \omega_{0, \pi]}(t)=\omega_{] 0, \varepsilon[}(t)+\omega_{](a-\varepsilon) / 2,(a+\varepsilon) / 2[}(t)+\omega_{]} a-\varepsilon, a+\varepsilon[ \\
&(t) \\
&+\omega_{]-\pi-(a / 2)-(\varepsilon / 2), \pi-(a / 2)+(\varepsilon / 2)[}(t)+\omega_{] \pi-(\varepsilon / 2), \pi]}(t) \\
&= \frac{1}{2}(\psi(t)+\psi(2 t))-\frac{i}{4} \psi(2 t-a)+\frac{i}{4} \psi(t-a) \\
& \quad+\frac{i}{4} \psi(2 t-2 \pi+a)+\frac{1}{2} \psi(2 t-2 \pi) .
\end{aligned}
$$

Similarly as in the proof of Proposition 1, the proof of the equality in $\left(\widehat{\mathrm{WS}}_{\mathbf{z}}\right)$ is trivially true for $n$ even. And for $n$ odd we have

$$
\begin{aligned}
& \frac{4^{n}}{i^{n-1}} \int_{\downarrow 0}^{\pi} \operatorname{Im} \varphi^{n}(t) \frac{d t}{2 \tan (t / 2)} \\
= & \int_{0}^{\pi}\left(-\psi^{n}(2 t-a)+\psi^{n}(t-a)+\psi^{n}(2 t-2 \pi+a)\right) \frac{d t}{2 \tan (t / 2)} \\
= & \int_{0}^{\pi} \psi^{n}(t-a) \cdot\left(\frac{-1}{4 \tan (t / 4)}+\frac{1}{2 \tan (t / 2)}+\frac{\tan (t / 4)}{4}\right) d t \\
= & 0 .
\end{aligned}
$$


[To obtain the equality in (8), decompose the integral in (7) into three in the obvious way, and perform the change of variables $t \mapsto t / 2$ in the first and $t \mapsto \pi-(t / 2)$ in the third. For the equality in (9), observe that the trigonometric expression in $(\ldots)$ in (8) vanishes identically.]

The final Proposition 3 of this paper is stated in more detail than necessary to complete the proof of our theorem. This is done for reference purposes, and for indicating the structure of our proof of its actually needed part iv) in the case (L).

Notation: $\mu_{k}(P):=\int x^{k} d P(x)$ whenever $\int|x|^{k} d P(x)<\infty$.

Proposition 3. Let $P$ be a probability measure on $\mathbf{R}$ satisfying (WS).

i) If $\mu_{1}(P)$ exists, then $\mu_{1}(P)=0$.

ii) If $\mu_{3}(P)$ exists, then $\mu_{1}(P)=\mu_{3}(P)=0$.

iii) If $P$ satisfies $(\mathrm{L})$ and if $\mu_{3}(P)$ exists, then $P$ is a Dirac measure or the following statement is true:

If $h$ and $a$ are as in $(\mathrm{L})$ and if $h$ is chosen maximal, then either $a=0$ $(\bmod h)$ or $a=h / 2(\bmod h)$.

iv) If $P$ satisfies $(\mathrm{C})$ or $(\mathrm{L})$, if $r \in \mathbf{N} \cup\{0\}$, and if $\mu_{2 r+1}(P)$ exists, then $\mu_{1}(P)=\mu_{3}(P)=\ldots=\mu_{2 r+1}(P)=0$.

Proof. We may assume that $P$ is not a Dirac measure. Also, in those cases where below the assumption (L) is made and $a, h$ are accordingly chosen, we may assume that $h$ is the span of $P$ [that is, $h$ is the maximal positive number such that $P$ is concentrated on $a+h \mathbf{Z}$ for some $a \in \mathbf{R}]$, that $h=1$, and that $a \in[0,1[$.

Let $\sigma^{2}$ denote the variance of $P$, whenever it is finite.

The four subpropositions will be proved in the order i), iii), iv), ii).

i) This follows easily from the weak law of large numbers.

iii) Assume that neither $a=0$ nor $a=1 / 2$. Put $\tilde{\mathbf{N}}:=\{n \in \mathbf{N}: n a \not \equiv 0(\bmod 1)\}$. Then, using the first order Edgeworth expansion theorem for the lattice case [2, page 56] or [4, page 213], we have for $\tilde{\mathbf{N}} \ni n \rightarrow \infty$

$$
\begin{aligned}
\frac{1}{2} & \left.\left.=P^{* n}(]-\infty, 0\right]\right) \quad[\text { by }(\mathrm{WS}) \text { and } n \in \tilde{\mathbf{N}}] \\
& =\frac{1}{2}+\frac{\mu_{3}}{\sigma^{3} 6 \sqrt{2 \pi n}}+\frac{n a-\lfloor n a\rfloor-1 / 2}{\sigma \sqrt{2 \pi n}}+o\left(\frac{1}{\sqrt{n}}\right) \quad \text { [by Edgeworth], }
\end{aligned}
$$

with $\lfloor x\rfloor$ denoting the integer part of $x$. This implies convergence of the sequence $\left(n a-\lfloor n a\rfloor-\frac{1}{2}: n \in \tilde{\mathbf{N}}\right)$. But this is impossible since the unrestricted sequence $\left(n a-\lfloor n a\rfloor-\frac{1}{2}: n \in \mathbf{N}\right)$ has at least three accumulation values, and at most one of them, namely $-1 / 2$, can be eliminated by restricting $n$ to $\tilde{\mathbf{N}}$.

iv) This will be proved in four steps.

iv.a) In the case where $P$ satisfies (C), our claim is proved in [6, page 103].

iv.b) Assume that $P$ satisfies (L) with $a=0$.

As in iv.a), the idea is to use a suitable Edgeworth expansion, in this case for $P^{* n}(]-\infty, x[)+\frac{1}{2} P^{* n}(\{x\})$ with $x=0$. Instead of stating, proving and applying such an expansion, it seems to be technically simpler to work with $\left(\widehat{\mathrm{WS}}_{\mathbf{Z}}\right)$ directly, which amounts to proving the said Edgeworth expansion in a very special case.

From i) we know that iv) is true for $r=0$. So, for an inductive proof, assume that $r \in \mathbf{N}$ and that iv) is true with $r-1$ in place of $r$. 
Let $\kappa_{1}, \ldots, \kappa_{2 r+1}$ denote the first $2 r+1$ cumulants of $P$. By the induction hypothesis, $\mu_{1}(P)=\mu_{3}(P)=\ldots=\mu_{2 r-1}(P)=0$, which is equivalent to

$$
\kappa_{1}=\kappa_{3}=\ldots=\kappa_{2 r-1}=0 .
$$

Choose $c \in] 0, \infty[$ such that

$$
|\varphi(t)| \leq 1-c t^{2} \quad(|t| \leq \pi)
$$

[To find such a constant $c$, use [9, page 11] and the fact that, for $\delta>0, \sup \{|\varphi(t)|$ : $\delta \leq t \leq \pi\}<1$.]

Choose a sequence of numbers $\left.\delta_{n} \in\right] 0, \pi[$ which tends to zero, on the one hand so quickly that

$$
\begin{aligned}
\lim _{n \rightarrow \infty} \delta_{n}^{4} \cdot n & =0, \\
\lim _{n \rightarrow \infty} \delta_{n}^{6 r+2} \cdot n^{r+2} & =0,
\end{aligned}
$$

but on the other hand so slowly that

$$
\lim _{n \rightarrow \infty}\left(\left(1-c \delta_{n}^{2}\right)^{n} \cdot \log \frac{\pi}{\delta_{n}}\right) \cdot n^{r-(1 / 2)}=0 .
$$

[For example, we may take $\delta_{n}=n^{-\alpha}$ for $n \in \mathbf{N}$, with any $\left.\alpha \in\right] 3 / 8,1 / 2[$.$] Now$

$$
\begin{aligned}
\int_{\delta_{n}}^{\pi}\left|\varphi^{n}(t)\right| \cdot \cot \frac{t}{2} d t & \leq \int_{\delta_{n}}^{\pi}\left(1-c \delta_{n}^{2}\right)^{n} \cdot \frac{2}{t} d t \quad[\text { by }(11) \text { and } \tan x \geq x] \\
& =o\left(n^{-(r-(1 / 2))}\right) \quad(n \rightarrow \infty) \quad[\text { by }(14)] .
\end{aligned}
$$

Combined with $\left(\widehat{\mathrm{WS}}_{\mathbf{Z}}\right)$, this yields, for $n \rightarrow \infty$,

$$
0=\int_{0}^{\delta_{n}} \operatorname{Im} \varphi^{n}(t) \cdot \cot \frac{t}{2} d t+o\left(n^{-(r-(1 / 2))}\right)
$$

Thus

$$
\lim _{n \rightarrow \infty} \int_{0}^{\tau_{n}} \psi_{n}(t) d t=0
$$

with

$$
\begin{aligned}
\tau_{n} & :=\delta_{n} \cdot \sqrt{n}, \\
\psi_{n}(t) & :=n^{r-(1 / 2)} \cdot \operatorname{Im} \varphi^{n}\left(\frac{t}{\sqrt{n}}\right) \cdot\left(\cot \frac{t}{2 \sqrt{n}}\right) \cdot \frac{1}{\sqrt{n}} \quad(t \in] 0, \infty[) .
\end{aligned}
$$

We now claim that

$$
\lim _{n \rightarrow \infty} \psi_{n}(t)=2 \cdot \kappa_{2 r+1} \frac{(-)^{r} t^{2 r}}{(2 r+1) !} \exp \left(-\sigma^{2} t^{2} / 2\right) \quad(t \in] 0, \infty[)
$$

and that

$$
\int_{0}^{\infty} \sup _{n \in \mathbf{N}}\left(\left|\psi_{n}(t)\right| \cdot 1_{] 0, \tau_{n}[}(t)\right) d t<\infty .
$$

Let us accept (16) and (17) for the moment. Then the dominated convergence theorem shows that the limit on the left-hand side of (15) is a nonzero multiple of $\kappa_{2 r+1}$. Hence $\kappa_{2 r+1}=0$, so that iv) is true for the present $r$.

iv.c) We now prove the relations (16) and (17) used in step iv.b) above. Note first that for $t \in] 0, \infty[$

$$
\lim _{n \rightarrow \infty} \frac{t}{\sqrt{n}} \cdot \cot \frac{t}{2 \sqrt{n}}=2, \quad\left|\frac{t}{\sqrt{n}} \cdot \cot \frac{t}{2 \sqrt{n}}\right| \cdot 1_{] 0, \tau_{n}[}(t) \leq 2 .
$$


Further, using $\lim _{n \rightarrow \infty} \delta_{n}=0$ and simple Taylor expansion arguments, we get the following asymptotic relations, valid for $n \rightarrow \infty$ uniformly in $t \in\left[0, \tau_{n}\right]$ :

$$
\begin{aligned}
& \frac{n^{r-(1 / 2)}}{t} \operatorname{Im} \varphi^{n}\left(\frac{t}{\sqrt{n}}\right) \\
= & \frac{n^{r-(1 / 2)}}{t} \operatorname{Im} \exp \left(n \cdot \log \varphi\left(\frac{t}{\sqrt{n}}\right)\right) \\
= & \frac{n^{r-(1 / 2)}}{t} \operatorname{Im} \exp \left(n \cdot \sum_{l=1}^{r} \frac{\kappa_{2 l}}{(2 l) !}\left(\frac{i t}{\sqrt{n}}\right)^{2 l}+n \cdot \frac{\kappa_{2 r+1}}{(2 r+1) !}\left(\frac{i t}{\sqrt{n}}\right)^{2 r+1}\right. \\
& \left.+n \cdot o\left(\frac{t}{\sqrt{n}}\right)^{2 r+1}\right) \\
= & \exp \left(\sum_{l=1}^{r} \frac{\kappa_{2 l}(-)^{l} t^{2 l}}{(2 l) ! n^{l-1}}\right) \cdot \frac{n^{r-(1 / 2)}}{t} \cdot\left(\sin \left(\frac{\kappa_{2 r+1}}{(2 r+1) !} \frac{(-)^{r} t^{2 r+1}}{n^{r-(1 / 2)}}\right)+o\left(\frac{t^{2 r+1}}{n^{r-(1 / 2)}}\right)\right) \\
= & \exp \left(-\sigma^{2} t^{2} / 2\right) \cdot\left(\exp O\left(t^{4} / n\right)\right) \cdot\left(\frac{\kappa_{2 r+1}}{(2 r+1) !}(-)^{r} t^{2 r}+O\left(\frac{t^{6 r+2}}{n^{2 r-1}}\right)+o\left(t^{2 r}\right)\right) .
\end{aligned}
$$

[When checking this, keep in mind that $t \in\left[0, \tau_{n}\right]$ forces $t / \sqrt{n} \rightarrow 0$ for $n \rightarrow$ $\infty$. For the 2nd equality, remember (10). For the 3rd equality, observe that, for bounded $\zeta \in \mathbf{C}, e^{i x+\zeta}=e^{i x}+O(\zeta)$ uniformly for $x \in \mathbf{R}$, and that boundedness of $n \cdot o(t / \sqrt{n})^{2 r+1}$ follows from (13).]

Now using (12) and (13), we deduce that, uniformly in $t \in\left[0, \tau_{n}\right]$, each of the two $O(\ldots)$ symbols above is a $o(1)$, and hence

$$
t^{-1} n^{r-(1 / 2)} \operatorname{Im} \varphi^{n}\left(\frac{t}{\sqrt{n}}\right)=\frac{1}{2} \cdot(\text { R.H.S. of }(16))+\exp \left(-\sigma^{2} t^{2} / 2\right) \cdot o\left(1+t^{2 r}\right) .
$$

This and (18) easily yield (16) and (17).

iv.d) We now consider the only remaining case that $P$ satisfies (L) with $a \neq 0$. By i), we may restrict attention to $r \geq 1$. But then iii) implies that $a=1 / 2$. Now consider the probability measure $P^{* 2}$. It satisfies (WS) and (L), the latter with $h=1$ and $a=0$. Hence, by iv.b), existence of $\mu_{2 r+1}(P)$ implies

$$
\int x^{2 k+1} d P^{* 2}(x)=0 \quad(k=0, \ldots, r) .
$$

This is easily seen to be equivalent to (19) with $P$ replacing $P^{* 2}$. [Use Lemma 4.1 of Staudte \& Tata (1970). Alternatively, express both conditions in terms of the cumulants of $P$.]

ii) The case where $P$ satisfies (L) is contained in part iv), already proved above. So we may assume that (L) is not true. Then, using i), an application of Esseen's uniform first order Edgeworth expansion theorem for non-lattice distributions (see [2, page 49] or [4, page 210]) easily yields that

$$
\begin{aligned}
P^{* n}(]-\infty, 0[)+\frac{1}{2} P^{* n}(\{0\}) & \left.\left.\left.\left.=\frac{1}{2}\left(P^{* n}(]-\infty, 0\right]\right)+\lim _{x \rightarrow 0-} P^{* n}(]-\infty, x\right]\right)\right) \\
& =\frac{1}{2}+\frac{\mu_{3}(P)}{\sigma^{3} 6 \sqrt{2 \pi n}}+o\left(\frac{1}{\sqrt{n}}\right) .
\end{aligned}
$$

Hence (WS) forces that $\mu_{3}(P)=0$. 


\section{ACKNOWLEDGEMENT}

I thank the referee for his careful reading of the paper.

\section{REFERENCES}

1. Donoghue, W.F. (1969). Distributions and Fourier Transforms. Academic, N.Y.

2. Esseen, C.-G. (1944). Fourier analysis of distribution functions. A mathematical study of the Laplace-Gaussian law. Acta Math. 77, 1-125. MR 7:312a

3. Feller, W. (1971). An Introduction to Probability and Its Applications, Vol. 2. Second Edition. Wiley, New York. MR 42:5292

4. Gnedenko, B.V. and Kolmogorov, A.N. (1968). Limit distributions for sums of independent random variables. Addison- Wesley, Reading. MR 38:1722

5. Gurland, J. (1948). Inversion formulae for the distribution of ratios. Ann. Math. Statist. 19, 228- 237. MR 9:582a

6. Mattner, L. (1995). Mean unbiased medians, median unbiased means, and symmetric random walks. Mathematical Methods of Statistics 4, 99-105. MR 96c:62043

7. Mattner, L. (1997). Acknowledgement of priority. Mathematical Methods of Statistics 6, 517. CMP 98:12

8. Mattner, L. (199?). Gurland's Fourier inversion for distribution functions. (In preparation.)

9. Petrov, V. V. (1975). Sums of independent random variables. Springer, Berlin. MR 52:9335

10. Pollak, M. (1973). On equal distributions. Ann. Statist. 1, 180-182. MR 48:9914

11. Ramachandran, B. (1997). Characteristic functions with some powers real-III. Statistics $\&$ Probability Letters 34, 33-36. CMP 97:15

12. Staudte, R.G. and Tata, M.N. (1970). Complex roots of real characteristic functions. Proc. Amer. Math. Soc. 25, 238-246. MR 41:9323

Universität Hamburg, Institut für Mathematische Stochastik, Bundesstr. 55, D20146 Hamburg, Germany

E-mail address: mattner@math.uni-hamburg.de 